\section{Gastos da Secretaria Municipal de Saúde do Rio de Janeiro, Brasil, com medicamentos: uma análise do período 2002-2011}

\author{
Expenditures on medicines by the Rio de Janeiro \\ Municipal Health Department, Brazil, 2002-2011
}

\author{
Gastos en medicamentos del Departamento de \\ Salud Municipal de Río de Janeiro, Brasil: un \\ análisis del período 2002-2011
}

\author{
1 Instituto de Medicina \\ Social, Universidade do \\ Estado do Rio de Janeiro, \\ Rio de Janeiro, Brasil. \\ 2 Escola Nacional de Saúde \\ Pública Sergio Arouca, \\ Fundação Oswaldo Cruz, \\ Rio de Janeiro, Brasil. \\ Correspondência \\ R. M. Silva \\ Núcleo de Assistência \\ Farmacêutica, Escola \\ Nacional de Saúde Pública \\ Sergio Arouca, Fundação \\ Oswaldo Cruz. \\ Rua Leopoldo Bulhões 1480 \\ Rio de Janeiro, RJ \\ 21041-210, Brasil. \\ rondineli.mendes@gmail.com
}

\begin{abstract}
This study analyzed time trends in the purchase of medicines by the Rio de Janeiro Municipal Health Department, Brazil, from 2002 to 2011, analyzing data on total annual amounts and unit prices. Annual expenditure per inhabitant and expenditures on medicines as a proportion of total municipal health spending were calculated. Expenditures were adjusted according to the Expanded Consumer Price Index to compare them to 2011. Medicines were classified according to the predominant place of use (in-hospital or primary care) and the WHO Anatomical Therapeutic Chemical classification. Adjusted total expenditures totaled some BRL 575 million, with a decline over the period. Antimicrobials and medicines for the cardiovascular and nervous systems accounted for 55\% of total expenditures. There was a shift from in-hospital spending on medicines to those used predominantly in primary care. The analysis can contribute to healthcare management and emphasizes the need to reorient the municipality's policy for medicines.
\end{abstract}

Health Expenditures; Health Management; Pharmaceutical Services; Drugs
Rondineli Mendes da Silva 1,2 Rosângela Caetano ${ }^{1}$

\section{Resumo}

O estudo analisou o comportamento histórico das compras de medicamentos da Secretaria Municipal do Rio de Janeiro, Brasil, entre 20022011. Utilizaram-se dados financeiros sobre quantidade anual total adquirida e preço unitário. Calcularam-se o gasto anual por habitante e a proporção das despesas com medicamentos frente aos gastos municipais em saúde. Os gastos foram ajustados pelo Índice de Preços ao Consumidor Amplo para compará-los a 2011. Os medicamentos foram classificados, segundo ambiente de predomínio de uso, em hospitalar ou atenção primária e pelas classes do sistema anatômico-terapêutico-químico da Organização Mundial da Saúde. Os gastos totais ajustados foram de cerca de R\$ 575 milhões, com redução ao longo do intervalo analisado. Medicamentos anti-infecciosos e vinculados aos sistemas cardiovascular e nervoso responderam por 55\% dos gastos totais. Houve transição dos gastos com medicamentos do ambiente hospitalar para aqueles de uso predominante na atenção primária em saúde. A análise pode potencializar a gestão e sinaliza para necessidade de correção de rumos na política municipal de medicamentos.

Gastos em Saúde; Gestão em Saúde; Assistência Farmacêutica; Medicamentos 


\section{Introdução}

Um tema central para o desenvolvimento das ações de Assistência Farmacêutica refere-se ao seu financiamento. Nesse sentido, a Política Nacional de Medicamentos 1 de 1998 estabeleceu a diretriz de reorientação da assistência farmacêutica, articulada em três pontos: garantia de recursos pelas três esferas para distribuição direta ou descentralizada, descentralização plena da aquisição e distribuição de medicamentos, e financiamento específico para os medicamentos da atenção básica.

O movimento descentralizador no financiamento da assistência farmacêutica, especificamente voltado ao contexto da atenção primária em saúde, é inaugurado pela vigência da Portaria GM/MS no 176/1999 2, que define um incentivo à assistência farmacêutica básica, cabendo ao Ministério da Saúde a gestão central dos programas estratégicos. Estabeleceu-se o repasse financeiro direto do fundo federal aos fundos estaduais e/ou municipais, permitindo a estes entes governamentais maior autonomia na aquisição de medicamentos 2 .

Naquele momento, o financiamento da assistência farmacêutica envolvia um recurso mínimo de R\$ 2,00 por habitante/ano. Em 2005 e 2007, verificaram-se aumentos no aporte de recursos 3,4,5 e um reforço no papel do município como gestor da aquisição de medicamentos relacionados à atenção básica, em conformidade à Política Nacional de Medicamentos. O financiamento mínimo atual 6 por habitante/ano, somando-se as contrapartidas dos três entes federativos, é de $\mathrm{R} \$$ 8,82, com o Ministério da Saúde contribuindo com $\mathrm{R} \$ 5,10$ e estados e municípios participando, cada um, com $\mathrm{R} \$ 1,86$.

No presente, os recursos de financiamento do bloco da assistência farmacêutica estão categorizados em três componentes: Básico, Especializado e Estratégico, onde os três gestores do SUS, dependendo da especificidade de cada um dos componentes, participam financiando através da aquisição direta ou por repasse de contrapartidas financeiras 7. O Componente Básico da Assistência Farmacêutica é destinado à aquisição de medicamentos e insumos no âmbito da atenção básica em saúde, estando vinculado a um elenco de referência nacional de medicamentos e insumos complementares. A Relação Nacional de Medicamentos Essenciais (RENAME) 8, ainda é a base de organização da lista em vigor.

ORiodeJaneiro,segundamaiorcapitaldopaís, possuía 6.355.949 habitantes em 2011 (Instituto Brasileiro de Geografia e Estatística. Estimativa da População. http://www.ibge.gov.br/home/ presidencia/noticias_impressao.php?id_noti cia=1961, acessado em 15/Jul/2012). A Secretaria Municipal de Saúde do Rio de Janeiro (SMS-RJ) responde por uma rede de mais de 23 hospitais próprios com diferentes graus de complexidade e 3.329 leitos (Cadastro Nacional dos Estabelecimentos de Saúde: http://cnes.datasus.gov.br/Lista_Es_Mu nicipio.asp? VEstado $=33 \&$ VCodMunicipio $=$ $330455 \&$ NomeEstado=RIO $\% 20$ DE $\% 20 J A$ NEIRO, acessado em 23/Jul/2012). Possui ainda 228 unidades básicas, tendo passado por uma expansão significativa de cobertura da Estratégia Saúde da Família (ESF) a partir de 20099.

A dotação orçamentária para a área de assistência farmacêutica computa os recursos originalmente do tesouro municipal e as transferências das esferas federal e estadual. A programação anual de medicamentos é realizada pela gerência central de assistência farmacêutica, utilizando predominantemente o método do consumo histórico, com os processos de aquisição sendo registrados em um sistema de informações específico.

Na última década, a rede municipal de serviços de saúde sofreu algumas mudanças que podem ter impactado no perfil de aquisições de medicamentos e outros insumos da SMS-RJ. Como antiga capital do país, o Rio de Janeiro possuía um grande número de unidades federais de saúde, sobretudo hospitalares, que sofreram um processo de municipalização a partir de 199910. Em 2005, ocorreu intervenção federal em quatro hospitais federais anteriormente municipalizados e dois hospitais municipais próprios, que foram justificadas, através do Decreto Presidencial no 5.392/2005 11, como devidas a um "estado de calamidade pública” decorrente da repetida insuficiência de insumos e, principalmente, de recursos humanos. Ainda neste mesmo ano, o Supremo Tribunal Federal considerou o processo de intervenção nos hospitais próprios como inconstitucional e estas unidades voltaram à gestão da SMS-RJ. No entanto, as quatro unidades hospitalares originalmente federais retornaram, em definitivo, para a administração federal 10 .

As mudanças acima referidas e as significativas alterações ocorridas na política de financiamento da assistência farmacêutica no país, bem como o porte do município, a complexa rede de serviços de saúde sob sua alçada e organização relativamente estruturada de sua assistência farmacêutica, assinalam aspectos que tornam interessante estudar o perfil de aquisições de medicamentos no Município do Rio de Janeiro. Assim, o trabalho objetivou analisar o comportamento histórico dos gastos com aquisição de medicamentos da SMS-RJ de 2002-2011, com foco na tendência desses gastos e em sua composição 
pelo lócus de utilização preferencial na rede de serviços.

\section{Metodologia}

O trabalho, de cunho exploratório-descritivo, foi estruturado com base nos dados de aquisição de medicamentos pela SMS-RJ durante o decênio 2002-2011, independente da origem inicial dos recursos dedicados às compras serem municipais ou de transferências estadual ou federal. As aquisições relacionadas a processos de judicialização foram excluídas das análises, em função de seu caráter irregular e, muitas vezes, de compra emergencial; as mesmas estão fora do banco de dados utilizado como fonte para a pesquisa, sendo gerenciadas e registradas em outro sistema peculiar.

Foram utilizados dados financeiros públicos de aquisição oriundos dos processos de empenho com medicamentos no período. O empenho representa o primeiro estágio de execução da despesa pública, não significando, contudo, que estes foram os valores efetivamente liquidados, executados quando da entrega e pagamento dos materiais fornecidos (Portal da Transparência do Governo Federal. http://www.portaldatrans parencia.gov.br/glossario/, acessado em 13/Fev/ 2013), que nos sistemas de dados da SMS não se encontram desagregados por itens adquiridos.

Os relatórios de compras foram extraídos do Sistema de Controle, Planejamento e Distribuição de Materiais (SISMED), que emite as ordens de entrega aos fornecedores, os quais, por sua vez, abastecem o almoxarifado farmacêutico central com medicamentos do Componente Básico da assistência farmacêutica 12. Embora públicos e divulgados no Diário Oficial do $\mathrm{Mu}$ nicípio, permissão de acesso aos dados foi obtida junto à Subsecretaria de Gestão da SMS-RJ.

Os relatórios extraídos do SISMED relativos ao período 2002-2011 foram diretamente trabalhados no aplicativo Microsoft Excel (Microsoft Corp., Estados Unidos), com organização de planilhas anuais contendo descrição dos medicamentos, quantidade total adquirida e preço unitário. Nestas planilhas, cada medicamento, com sua forma de apresentação, constitui um item individualizado, permitindo calcular o gasto específico para cada um destes.

Os medicamentos adquiridos foram categorizados segundo seu ambiente de maior consumo e uso, através de uma adaptação das convenções adotadas na RENAME 2010 8. As categorias convencionadas para este estudo foram: $(\mathrm{H})$ medicamentos destinados para uso predominantemente hospitalar, em pacientes internados ou am- bulatoriais que necessitam de atendimento especializado; (AP) medicamentos voltados para o atendimento ambulatorial na atenção primária; e, (H/AP) produtos com dupla carga de utilização, tanto hospitalar como atenção primária, não sendo possível definir predomínio de um ambiente de uso. Comparação por estas categorias mostra-se relevante pelo fato de a SMS-RJ possuir uma rede complexa de saúde e contar com um grande número de unidades e leitos hospitalares, que podem determinar demandas específicas.

Os medicamentos foram também categorizados de acordo com o Sistema de Classificação Anatômico-Terapêutico-Químico, ou ATC (sigla em inglês de Anatomical Therapeutic Chemical), até o terceiro nível (subgrupo terapêutico), a partir de consulta à página eletrônica da WHO Collaborating Centre for Drug Statistics Methodology 13.

Para os dados de população necessários às estimativas de gastos per capita, foram utilizadas as informações demográficas presentes na página eletrônica do Instituto Brasileiro de Geografia e Estatística (IBGE). A estimativa do gasto por habitante foi obtida pela divisão entre o volume de recursos dispendidos pela SMS-RJ com as aquisições de fármacos em um dado ano e a população informada pelo IBGE para o mesmo.

Os gastos com aquisição de medicamentos foram ainda avaliados como percentual dos gastos municipais em saúde, a partir de valores relativos ao Rio de Janeiro, obtidos em consulta à página eletrônica do Sistema de Informações sobre Orçamentos Públicos em Saúde (SIOPS. http://www.siosp.datasus.gov.br, acessado em 15/Out/2012).

Os gastos anuais são apresentados em valores correntes e, considerando a extensão do período analisado e a importância da atualização monetária dos valores para avaliação de tendências, foram também ajustados aplicando-se o Índice de Preços ao Consumidor Amplo (IPCA), permitindo compará-los aos valores de 2011, utilizando informações obtidas do Instituto de Pesquisas Econômicas Aplicadas (IPEA. Ipeadata Macroeconômico. http://www.ipeadata.gov.br/, acessado em 15/Jul/ 2012). A opção pelo uso desse deflator tomou por base a Lei no 10.742/2003 14, que estabeleceu as normas de regulação do setor farmacêutico e define este índice, calculado pelo IBGE, para fins do ajuste nos preços dos medicamentos.

\section{Resultados}

A estimativa de gastos correntes per capita, tomando por base a população do município para cada ano do estudo (Tabela 1), mostrouse oscilante até 2009, com clara tendência de 
Tabela 1

Itens, unidades farmacêuticas, gastos totais por habitante e porcentagem das despesas com medicamentos sobre despesas totais de saúde com aquisição de medicamentos da Secretaria Municipal de Saúde do Rio de Janeiro, Brasil, no período 2002-2011, em valores correntes e corrigidos.

\begin{tabular}{|c|c|c|c|c|}
\hline Ano & $\begin{array}{l}\text { Total de itens } \\
\text { adquiridos/ano }\end{array}$ & $\begin{array}{c}\text { Número de unidades } \\
\text { farmacêuticas adquiridas }\end{array}$ & $\begin{array}{c}\text { Gasto total corrente } \\
\text { por habitante }(R \$)\end{array}$ & $\begin{array}{c}\text { Despesa } \\
\text { corrente com } \\
\text { medicamento } \\
\text { (\% dos gastos } \\
\text { municipais em } \\
\text { saúde) }\end{array}$ \\
\hline 2002 & 488 & 190.292 .372 & 7,91 & 3,82 \\
\hline 2003 & 495 & 223.451 .856 & 7,18 & 3,04 \\
\hline 2004 & 479 & 373.016 .558 & 8,32 & 3,44 \\
\hline 2005 & 389 & 271.741 .387 & 5,65 & 2,86 \\
\hline 2006 & 424 & 462.850 .464 & 8,1 & 4,46 \\
\hline 2007 & 389 & 667.871 .372 & 7,43 & 2,63 \\
\hline 2008 & 400 & 433.623 .108 & 6,8 & 2,16 \\
\hline 2009 & 325 & 506.754 .926 & 5,86 & 1,79 \\
\hline 2010 & 347 & 751.794 .865 & 7,46 & 1,88 \\
\hline 2011 & 452 & 623.965 .693 & 8,83 & 1,82 \\
\hline Média (DP) & $419(59)$ & $450.536 .260(191.177 .177)$ & $7,35(1,00)$ & $2,79(1,00)$ \\
\hline Total & 4.188 & 4.505 .362 .601 & - & - \\
\hline
\end{tabular}

DP: desvio-padrão.

redução entre 2006 e este ano. A partir daí, retoma o crescimento e atinge, em 2011, o maior valor de período ( $\mathrm{R} \$ 8,83 /$ habitante). Este padrão se mantém mesmo quando se excluem os gastos com antineoplásicos, que fizeram parte do rol de aquisições da SMS-RJ durante os primeiros três anos do período examinado, em função da presença, na esfera municipal, de hospitais da rede federal que se encontravam naquele momento na gestão local.

As despesas correntes com medicamentos como proporção dos gastos municipais em saúde foram, em média, de 2,8\%. A maior proporção foi atingida em 2006 (4,5\%) e a menor em 2009 $(1,8 \%)$. O percentual relativo a $2011(1,8 \%)$ corresponde a $40 \%$ da presente em 2006 , que representa o ano com maior proporção de gastos no período investigado.

O número de itens adquiridos, entendido item como medicamento com cada uma de suas formas de apresentação, flutuou bastante, variando entre 325 e 495 (média de 419). O ano de 2003 representou o período de maior diversidade de itens comprados; apenas em 2005, 2009 e 2010, houve aquisição inferior a 400 itens (Tabela 1).

$\mathrm{O}$ número médio de unidades farmacêuticas, independente da apresentação do produto, foi de 4.505.362.601, novamente com significa- tiva variação anual. Em 2010, foram adquiridos mais de 750 milhões de unidades farmacêuticas ( $16,7 \%$ do total), apesar de este ano ter contribuído com $8,7 \%$ do gasto total.

$\mathrm{O}$ valor total de recursos financeiros gastos pela SMS-RJ com a compra de medicamentos no período de estudo foi de aproximadamente R\$ 575,5 milhões, com média anual de R\$ 57,5 milhões, em valores corrigidos pelo IPCA a 2011 (Tabela 2).

São percebidas também grandes variações nos gastos anuais entre 2002-2011. Embora com algumas oscilações, houve tendência de queda no período, só revertida a partir de 2010. O ano de 2002 correspondeu aos maiores dispêndios com R\$ 77.315.228,39 (13,4\% do total), seguido de 2004 com cerca de R $\$ 71$ milhões (12,3\%); 2009 representou o menor valor de gasto $(7,1 \%)$. Os gastos ajustados relativos aos anos entre 2002 a 2006 corresponderam a $56,2 \%$ do total.

Segundo o lócus principal de uso, verificou-se que medicamentos de uso predominantemente ambulatorial e na atenção primária corresponderam, em média, a cerca de metade dos gastos corrigidos do decênio examinado, variando entre 35\% (2002) e 68\% (2010) do total. Embora com oscilações, há uma propensão de crescimento dos gastos neste lócus de atenção. Em contra- 
Tabela 2

Distribuição dos gastos com aquisição de medicamentos da Secretaria Municipal de Saúde do Rio de Janeiro, Brasil, no período 2002-2011, segundo o ambiente principal de uso, em valores correntes e corrigidos (em R\$).

\begin{tabular}{|c|c|c|c|c|c|c|c|c|c|c|c|}
\hline \multirow[t]{2}{*}{ Ano } & \multicolumn{3}{|c|}{ AP } & \multicolumn{3}{|c|}{ H/AP } & \multicolumn{3}{|c|}{$\mathrm{H}$} & \multicolumn{2}{|c|}{ Total } \\
\hline & $\begin{array}{c}\text { Gasto } \\
\text { corrente }\end{array}$ & $\begin{array}{c}\text { Gasto } \\
\text { corrigido * }\end{array}$ & $\begin{array}{l}\% \text { do } \\
\text { total } \\
\text { anual }\end{array}$ & $\begin{array}{c}\text { Gasto } \\
\text { corrente }\end{array}$ & $\begin{array}{c}\text { Gasto } \\
\text { corrigido * }\end{array}$ & $\begin{array}{l}\% \text { do } \\
\text { total } \\
\text { anual }\end{array}$ & $\begin{array}{c}\text { Gasto } \\
\text { corrente }\end{array}$ & $\begin{array}{c}\text { Gasto } \\
\text { corrigido * }\end{array}$ & $\begin{array}{l}\% \text { do } \\
\text { total } \\
\text { anual }\end{array}$ & $\begin{array}{c}\text { Gasto } \\
\text { corrente }\end{array}$ & $\begin{array}{c}\text { Gasto } \\
\text { corrigido * }\end{array}$ \\
\hline 2002 & $16.332 .689,65$ & $26.889 .894,82$ & 34,8 & $2.975 .028,01$ & $4.898 .041,41$ & 6,3 & $27.652 .883,68$ & $45.527 .292,16$ & 58,9 & $46.960 .601,34$ & $77.315 .228,39$ \\
\hline 2003 & $16.369 .961,94$ & $24.737 .273,39$ & 38,2 & $2.096 .257,63$ & $3.167 .734,80$ & 4,9 & $24.441 .180,50$ & $36.933 .999,37$ & 57,0 & $42.907 .400,07$ & $64.839 .007,55$ \\
\hline 2004 & $26.473 .142,22$ & $37.265 .555,81$ & 52,6 & $1.732 .890,90$ & $2.439 .345,58$ & 3,4 & $22.129 .239,88$ & $31.150 .757,14$ & 44,0 & $50.335 .273,00$ & $70.855 .658,53$ \\
\hline 2005 & $16.361 .447,81$ & $21.820 .545,53$ & 47,5 & $1.643 .664,95$ & $2.192 .083,88$ & 4,8 & $16.418 .600,05$ & $21.896 .766,96$ & 47,7 & $34.423 .712,82$ & $45.909 .396,37$ \\
\hline 2006 & $25.399 .250,77$ & $32.855 .351,16$ & 51,1 & $2.609 .747,50$ & $3.375 .854,32$ & 5,2 & $21.707 .346,12$ & $28.079 .666,05$ & 43,7 & $49.716 .344,38$ & $64.310 .871,52$ \\
\hline 2007 & $28.300 .855,58$ & $35.075 .921,46$ & 62,5 & $2.713 .683,20$ & $3.363 .323,71$ & 6,0 & $14.271 .412,38$ & $17.687 .908,35$ & 31,5 & $45.285 .951,15$ & $56.127 .153,52$ \\
\hline 2008 & $19.472 .037,12$ & $22.821 .308,23$ & 46,5 & $3.202 .910,71$ & $3.753 .824,63$ & 7,7 & $19.189 .952,27$ & $22.490 .703,61$ & 45,8 & $41.864 .900,10$ & $49.065 .836,47$ \\
\hline 2009 & $22.287 .016,44$ & $25.060 .419,90$ & 61,5 & $2.565 .041,90$ & $2.884 .236,54$ & 7,1 & $11.407 .523,87$ & $12.827 .079,79$ & 31,5 & $36.259 .582,21$ & $40.771 .736,23$ \\
\hline 2010 & $31.928 .195,61$ & $33.946 .057,57$ & 67,7 & $3.206 .204,74$ & $3.408 .836,87$ & 6,8 & $12.007 .904,27$ & $12.766 .803,82$ & 25,5 & $47.142 .304,61$ & $50.121 .698,26$ \\
\hline 2011 & $31.835 .137,67$ & $31.835 .137,67$ & 56,7 & $6.179 .107,17$ & $6.179 .107,17$ & 11,0 & $18.140 .233,51$ & $18.140 .233,51$ & 32,3 & $56.154 .478,35$ & $56.154 .478,35$ \\
\hline Total & $234.759 .734,81$ & $292.307 .465,53$ & 50,8 & $28.924 .536,71$ & $35.662 .388,92$ & 6,2 & $187.366 .276,52$ & $247.501 .210,75$ & 43,0 & $451.050 .548,03$ & $575.471 .065,20$ \\
\hline
\end{tabular}

AP: medicamentos voltados para o atendimento ambulatorial na atenção primária; H: medicamentos destinados para uso predominantemente hospitalar, em pacientes internados ou ambulatoriais que necessitam de atendimento especializado; H/AP: produtos com dupla carga de utilização, tanto hospitalar como atenção primária, não sendo possível definir predomínio de um ambiente de uso.

* Gasto anual corrigido utilizando-se o Índice de Preços ao Consumidor Amplo, possibilitando comparação aos valores de 2011.

partida, há uma tendência inversa, de queda, em relação aos medicamentos de uso predominantemente hospitalar, mesmo com elevação ocorrida em 2011 (Tabela 2). Exceto por 2011, em todos os demais anos, medicamentos de uso misto na atenção primária e no ambiente hospitalar (H/AP) - corresponderam sempre a menos de $10 \%$ do total de gastos da SMS-RJ com aquisição de fármacos.

Três classes ATC correspondem a quase 55\% do total de gastos corrigidos no período: anti-infecciosos $(24,5 \%)$; sistema cardiovascular $(17,1 \%)$ e sistema nervoso (14,2\%) (Tabela 3). Exceto em 2007, quando foram suplantados pelos medicamentos relacionados ao sistema cardiovascular, anti-infecciosos corresponderam sempre a categoria ATC de maior gasto, chegando a atingir 29\% do total em 2005. O grupo relativo aos antineoplásicos foi item significativo de compras apenas no período 2002-2004, quando correspondeu em média a $8 \%$ dos gastos totais da SMS-RJ.

Os gastos com medicamentos fitoterápicos, não presentes na classificação ATC, só começaram a se constituir em objeto de compra a partir de 2009, mas se elevaram em mais de $1000 \%$ até 2011 .

Analisando-se o comportamento dos gastos proporcionais das três principais categorias ATC (Tabela 4) segundo o local predominante de uso, observa-se que, no caso dos medicamen- tos relativos ao sistema cardiovascular, o lócus é sempre, em todos os anos do período, a atenção primária. No caso daqueles pertencentes à categoria N (sistema nervoso), o predomínio dos gastos, para o período agregado, corresponde a atenção primária em saúde em 55,8\%; observase também uma tendência de queda progressiva destes no ambiente hospitalar. Quanto aos antiinfecciosos, as oscilações ano a ano dos gastos, em valores corrigidos, não permitem estabelecer um ambiente predominante de utilização no período coberto pela análise.

Uma observação mais detalhada dos dez maiores itens de gastos totais mostrou que, nos dispêndios de 2002, prevaleciam gastos com medicamentos de uso predominante no ambiente hospitalar (6 itens, correspondendo a $68,7 \%$ dos $\mathrm{R} \$ 15.790 .763,06$ gastos reais deflacionados), enquanto existiam apenas dois produtos de uso na atenção básica (14,7\% dos gastos). Mesma avaliação para 2011 apresentou comportamento inverso, dominando entre os 10 maiores itens de gasto, medicamentos de uso predominante na atenção básica (6 itens, 52,5\% do total de R $\$ 7.943 .606,70$ ) sobre aqueles de utilização prevalente no âmbito hospitalar (apenas 2 itens, 11,4\% desse subtotal de gastos).

Observa-se o importante papel nos gastos com um único fármaco - cloridrato de hidralazina, na apresentação de comprimidos de $25 \mathrm{mg}$ - 
Tabela 3

Gastos totais com aquisição de medicamentos da Secretaria Municipal de saúde do Rio de Janeiro, Brasil, no período 2002-2011, segundo primeiro nível da classificação ATC, em valores corrigidos * (em R\$).

\begin{tabular}{|c|c|c|c|c|c|c|}
\hline Grupos terapêuticos ou farmacológicos & 2002 & 2003 & 2004 & 2005 & 2006 & 2007 \\
\hline Trato alimentar e metabolismo (A) & $5.664 .245,62$ & $4.357 .680,26$ & $6.217 .873,49$ & $4.080 .063,65$ & $6.610 .699,13$ & $5.835 .333,44$ \\
\hline Sangue e sistema hematopoiético (B) & $9.155 .999,21$ & $6.743 .336,44$ & $5.638 .837,94$ & $3.961 .079,40$ & $5.694 .154,13$ & $7.154 .681,57$ \\
\hline Sistema cardiovascular (C) & $7.461 .943,95$ & 7.087.109,30 & $15.112 .185,20$ & $9.333 .739,59$ & $12.179 .824,73$ & $13.209 .781,03$ \\
\hline Dermatológico (D) & $4.855 .352,26$ & $3.682 .246,45$ & $2.325 .793,63$ & $1.447 .912,26$ & $3.772 .547,02$ & $3.222 .256,00$ \\
\hline Sistema genito-urinário e hormônios sexuais (G) & $1.103 .859,59$ & $1.471 .004,75$ & $1.566 .547,68$ & $612.288,59$ & $805.295,93$ & $769.560,53$ \\
\hline Preparações hormonais sistêmicas $(H)$ & $1.786 .666,85$ & $1.030 .835,69$ & $1.477 .506,60$ & $851.128,22$ & $1.599 .700,54$ & $925.466,85$ \\
\hline Anti-infecciosos $(\mathrm{J})$ & $20.854 .296,34$ & $16.543 .416,90$ & $16.858 .597,37$ & $13.574 .740,60$ & $16.728 .515,98$ & $12.014 .961,76$ \\
\hline Antineoplásico (L) & $5.749 .764,75$ & $6.168 .221,18$ & $4.932 .213,31$ & $15.997,89$ & $20.357,38$ & $16.879,68$ \\
\hline Sistema músculo esquelético (M) & $3.634 .754,17$ & $3.532 .781,67$ & $1.961 .754,19$ & $1.449 .775,65$ & $2.527 .950,42$ & $1.163 .201,28$ \\
\hline Sistema nervoso (N) & $10.543 .891,48$ & $8.858 .425,49$ & $8.094 .609,89$ & $6.802 .660,89$ & $9.497 .234,30$ & $6.911 .559,95$ \\
\hline Antiparasitários (P) & $525.012,28$ & $341.284,29$ & $521.290,66$ & $316.561,32$ & $742.215,60$ & $607.845,68$ \\
\hline Sistema respiratório $(\mathrm{R})$ & $2.760 .462,03$ & $2.087 .269,96$ & $3.758 .549,91$ & $2.116 .174,00$ & $3.042 .708,82$ & $3.192 .042,33$ \\
\hline Órgãos sensores (S) & $525.323,96$ & $718.493,40$ & $554.377,79$ & $287.398,38$ & $398.467,35$ & $245.550,13$ \\
\hline Vários (V) & $2.693 .655,90$ & $2.216 .901,76$ & $1.835 .520,88$ & $1.059 .875,92$ & $691.200,22$ & $858.033,30$ \\
\hline Sem ATC (fitoterápico) & 0,00 & 0,00 & 0,00 & 0,00 & 0,00 & 0,00 \\
\hline Total & $77.315 .228,39$ & $64.839 .007,55$ & $70.855 .658,54$ & $45.909 .396,36$ & $64.310 .871,54$ & $56.127 .153,54$ \\
\hline Grupos terapêuticos ou farmacológicos & 2008 & 2009 & 2010 & 2011 & Gasto total & \\
\hline Trato alimentar e metabolismo (A) & $4.546 .057,52$ & $3.335 .222,52$ & $3.236 .947,09$ & $3.848 .352,24$ & $47.732 .474,97$ & \\
\hline Sangue e sistema hematopoiético (B) & $10.086 .572,74$ & $6.181 .764,03$ & $6.666 .073,11$ & $8.877 .596,30$ & $70.160 .094,88$ & \\
\hline Sistema cardiovascular (C) & $7.366 .165,38$ & $9.110 .998,36$ & $8.452 .259,38$ & $9.367 .884,96$ & $98.681 .891,89$ & \\
\hline Dermatológico (D) & $2.530 .045,12$ & $2.241 .269,48$ & $3.482 .361,90$ & $4.970 .819,41$ & $32.530 .603,53$ & \\
\hline Sistema genito-urinário e hormônios sexuais (G) & $589.567,26$ & $457.326,00$ & $1.173 .265,35$ & $826.036,84$ & $9.374 .752,52$ & \\
\hline Preparações hormonais sistêmicas $(H)$ & $717.786,36$ & $625.962,47$ & $716.313,52$ & $667.226,58$ & $10.398 .593,68$ & \\
\hline Anti-infecciosos $(\mathrm{J})$ & $10.289 .342,93$ & $10.040 .670,73$ & $11.580 .312,65$ & $10.527 .451,68$ & $139.012 .306,94$ & \\
\hline Antineoplásico (L) & $9.844,83$ & $3.538,95$ & $10.546,94$ & $8.514,30$ & $16.935 .879,22$ & \\
\hline Sistema músculo esquelético (M) & $990.598,73$ & $474.135,06$ & $1.502 .359,04$ & $1.557 .564,03$ & $18.794 .874,24$ & \\
\hline Sistema nervoso $(\mathrm{N})$ & $6.967 .902,25$ & $4.241 .517,69$ & $6.589 .086,85$ & $7.649 .219,20$ & $76.156 .107,99$ & \\
\hline Antiparasitários (P) & $320.216,78$ & $205.049,90$ & $194.380,58$ & $611.427,12$ & $4.385 .284,21$ & \\
\hline Sistema respiratório $(\mathrm{R})$ & $2.300 .621,89$ & $2.372 .873,82$ & $2.149 .062,52$ & $3.642 .654,15$ & $27.422 .419,43$ & \\
\hline Órgãos sensores (S) & $271.555,11$ & $164.534,75$ & $1.449 .214,71$ & $1.059 .618,98$ & $5.674 .534,56$ & \\
\hline Vários (V) & $2.079 .559,58$ & $1.280 .635,12$ & $2.821 .480,93$ & $1.841 .906,79$ & $17.378 .770,42$ & \\
\hline Sem ATC (fitoterápico) & 0,00 & $36.237,34$ & $98.033,69$ & $698.205,77$ & $832.476,79$ & \\
\hline Total & $49.065 .836,48$ & $40.771 .736,23$ & $50.121 .698,27$ & $56.154 .478,35$ & $575.471 .065,26$ & \\
\hline
\end{tabular}

ATC: Anatomical Therapeutic Chemical.

* Gasto anual corrigido utilizando-se o Índice de Preços ao Consumidor Amplo, possibilitando comparação aos valores de 2011.

que, desde 2005, ocupa posição de destaque, figurando como o item de maior impacto nos dispêndios de 2007-2010 e como o segundo maior item em 2011.

\section{Discussão}

Vieira \& Mendes 15 destacam a necessidade de se desenvolver análises do comportamento dos gastos de medicamentos no SUS, pois estes insumos têm exercido um papel destacado nos gastos em saúde, principalmente em sistemas nacionais de acesso universal. 
Distribuição percentual dos gastos da Secretaria Municipal de Saúde do Rio de Janeiro, Brasil, com aquisição de medicamentos dos três principais grupos ATC, segundo o ambiente principal de uso, no período 2002-2011.

\begin{tabular}{lcccccccccc}
\hline \multicolumn{2}{c}{ Ano } & \multicolumn{2}{c}{ Anti-infecciosos (J) } & \multicolumn{2}{c}{ Sistema cardiovascular (C) } & \multicolumn{2}{c}{ Sistema nervoso (N) } \\
& AP & AP/H & H & AP & AP/H & H & AP & AP/H \\
\hline 2002 & 34,4 & 0,0 & 65,6 & 78,4 & 1,9 & 19,7 & 30,9 & 2,1 & 67,0 \\
2003 & 36,8 & 0,1 & 63,2 & 81,9 & 1,0 & 17,1 & 37,6 & 0,7 & 61,6 \\
2004 & 38,7 & 0,0 & 61,3 & 94,6 & 1,2 & 4,2 & 54,2 & 0,9 & 44,9 \\
2005 & 28,1 & 0,0 & 71,9 & 88,2 & 1,5 & 10,3 & 52,9 & 2,1 & 44,9 \\
2006 & 39,1 & 0,0 & 60,9 & 88,6 & 1,1 & 10,3 & 45,6 & 3,1 & 51,3 \\
2007 & 54,9 & 0,0 & 45,1 & 93,5 & 0,9 & 5,6 & 66,2 & 6,7 & 27,1 \\
2008 & 45,5 & 0,0 & 54,5 & 85,2 & 1,4 & 13,4 & 61,2 & 6,7 & 32,0 \\
2009 & 57,2 & 0,0 & 42,8 & 92,2 & 0,5 & 7,3 & 66,4 & 1,4 & 32,2 \\
2010 & 65,2 & 0,0 & 34,8 & 94,7 & 0,8 & 4,6 & 74,0 & 4,5 & 21,5 \\
2011 & 42,0 & 0,0 & 58,0 & 93,7 & 0,6 & 5,7 & 69,3 & 6,1 & 24,5 \\
Média (DP) & $44,2(11,5)$ & $0,0(0,0)$ & $55,8(11,5)$ & $89,1(5,7)$ & $1,1(0,4)$ & $9,8(5,4)$ & $55,8(14,3)$ & $3,4(2,4)$ & $40,7(15,8)$
\end{tabular}

AP: medicamentos voltados para o atendimento ambulatorial na atenção primária; ATC: Anatomical Therapeutic Chemical;

DP: desvio-padrão; H: medicamentos destinados para uso predominantemente hospitalar, em pacientes internados ou ambulatoriais que necessitam de atendimento especializado; H/AP: produtos com dupla carga de utilização, tanto

hospitalar como atenção primária, não sendo possível definir predomínio de um ambiente de uso.

Alguns trabalhos têm se dedicado a examinar os gastos com assistência farmacêutica no Brasil, mas eles focam predominantemente no comportamento dos gastos federais 16,17. Entretanto, com a descentralização administrativa das unidades de saúde do âmbito federal e estadual para os municípios, os serviços farmacêuticos também passaram a ser executados, em sua maioria, pela esfera municipal, sendo relevante examinar os gastos com medicamentos relacionados a este ente subnacional, sobretudo em face da pouca informação existente a respeito ${ }^{18}$.

A avaliação do comportamento dos gastos da SMS-RJ com aquisição de medicamentos identificou uma significativa oscilação no decênio (Tabela 1). A ocorrência de transição de uma assistência farmacêutica fortemente hospitalar para aquela mais vinculada à atenção primária pode ser um fator contribuidor para o comportamento flutuante identificado. Dois aspectos reforçam essa hipótese: a saída de diversos hospitais federais da esfera municipal, com seu retorno à administração federal a partir de 2005, e a progressiva expansão da atenção básica no município, na forma da ESF, nos últimos anos do período examinado.

Não houve expansão nos gastos da SMS-RJ; pelo contrário, o exame dos gastos corrigidos mostra tendência de redução no período analisado, ainda que nos últimos três anos tenha ocorrido pequeno aumento nestes. Mesmo assim, os valores desses anos ficam claramente abaixo daqueles que vinham existindo na primeira metade do decênio sob análise.

Estudo de caráter nacional recente, realizado pelo IPEA, também demonstrou flutuações no gasto público global com aquisição de medicamentos, entre 2005-2008, com crescimento modesto no período 19 . Sobre os gastos de cada esfera de governo com o elenco de medicamentos sob sua responsabilidade, o trabalho menciona que os municípios contribuíram nos gastos totais proporcionais em 2005 com 20,2\%; estas contribuições para 2006, 2007 e 2008 corresponderam a respectivamente $17,1 \%, 13,3 \%$ e $17,5 \%$, evidenciando uma redução relativa, ainda que pequena, da participação municipal.

Na SMS-RJ, 2002-2004 corresponderam ao intervalo temporal de maior gasto (37\% do total corrigido). Em 2005, verifica-se forte queda, coincidindo com fase de crise financeiro-orçamentária da SMS-RJ que contribuiu para o processo de intervenção no município e de refederalização dos hospitais já mencionado. Destacase ainda que 2009 foi o ano de menor dispêndio com aquisições diretas de medicamentos da SMS-RJ. Além de subjacente à troca de governo, o que pode ter agregado dificuldades de execução orçamentária, nele ocorreu uma alteração nos procedimentos licitatórios da SMS-RJ, que passaram de pregões presenciais a eletrônicos. Esse fato fragilizou as compras e o abastecimento, 
com ocorrência de inúmeros itens fracassados nas licitações.

Não é possível se afirmar também que a elevação identificada a partir de 2010 seja uma tendência de retomada do volume de gastos com medicamentos. Pode ser que este aumento seja tão somente pontual, o que apenas poderá ser mais bem definido a partir do acompanhamento e exame nos gastos futuros da SMS-RJ.

A despeito disso, esse período coincide com a expansão da rede de atenção primária municipal, decorrente principalmente do aumento de cobertura da ESF no Rio de Janeiro, que sai da ordem de pouco mais de $10 \%$, presente sem grandes alterações entre 2006 e 2009, para mais de $30 \%$ no início de 2012 . Esta expansão repercutiu no volume de procedimentos clínicos (DATASUS. Despesas em Saúde. http://www2.datasus.gov.br/ DATASUS/index.php?area=0202, acessado em 20/Fev/2013) - a produção aprovada de atendimentos englobados nos códigos "consultas/ atendimentos/acompanhamentos" subiu, entre 2010 e 2011, de 6.639.831 para 10.541.774 (incremento de $59 \%$ ) - e pode também ter impactado no consumo de medicamentos da SMS.

O gasto médio per capita da SMS-RJ com medicamentos no período, em valores correntes, foi de R \$ 7,35. De forma semelhante aos gastos anuais, observa-se variação nos gastos por habitante no decênio, com tendência decrescente entre 2006 e 2009, e recuperação nos dois últimos anos analisados, atingindo em 2011 maior valor entre os dez anos examinados ( $\mathrm{R} \$ 8,83$ ).

Para tal recuperação, além da expansão da rede municipal de atenção primária em saúde já mencionada, pode ter contribuído a Portaria no 4.217/2010 6, em vigor a partir de 2010, que eleva o financiamento mínimo do componente básico da assistência farmacêutica dos três entes federativos para R\$ 8,82 per capita/ano. Este valor global representa um aumento de $341 \%$ nos valores mínimos de aplicação por habitante/ano, considerando os R \$ 2,00 definidos pela Portaria 176/99 2, que primeiro estabeleceu valores mínimos para o financiamento da assistência farmacêutica básica. A expansão das contrapartidas municipais, considerando o período histórico de 1999-2010, representou uma evolução de 272\%, tomando por base a aplicação dos recursos mínimos por habitante legalmente instituídos.

Comparações diretas entre os gastos municipais per capita com os valores definidos pelas diversas portarias ministeriais que estabelecem os valores mínimos por habitante/ano 6,7 devem ser feitas com muita cautela, porque estas últimas têm por foco apenas o financiamento do componente básico da assistência farmacêutica e pactuações em instâncias de deliberação pelas
Comissões Intergestores Bipartite, envolvendo os estados e municípios, é possível a existência de valores superiores aos contidos nas normas do Ministério da Saúde.

Os valores por habitante gastos pela SMS$\mathrm{RJ}$ nos últimos dois anos do período analisado - respectivamente $\mathrm{R} \$ 7,46$ e $\mathrm{R} \$$ 8,83 - apontam que, no máximo, o gasto municipal em 2010 e 2011 se aproxima ou iguala ao valor mínimo por habitante estabelecido pela Portaria no 4.217/10 6 para o financiamento apenas da assistência farmacêutica básica, levando a suspeita de subfinanciamento da assistência farmacêutica como um todo nessa Secretaria.

Um elemento que precisa ser lembrado neste contexto é o Programa Farmácia Popular, desenvolvido pelo Ministério da Saúde a partir de 2004, com o objetivo de ampliar o acesso da população aos medicamentos essenciais, a baixos preços, através de subsídios da União 20. O Programa sofreu forte expansão a partir de 2006, com a entrada do setor privado. Em julho de 2012, existiam vinculados ao Programa Farmácia Popular 19.666 estabelecimentos comerciais, cobrindo 3.353 municípios, sendo 457 farmácias no município do Rio de Janeiro 21. O Programa Farmácia Popular, através de sua vertente Saúde Não Tem Preço, disponibiliza acesso, sem desembolso direto, a cerca de 20 medicamentos comuns ao elenco do componente básico da assistência farmacêutica, entre anti-hipertensivos, antiasmáticos, antidiabéticos. Segundo informações do Ministério da Saúde, obtidas por contato direto com o Sistema Eletrônico do Serviço de Informações ao Cidadão (e-SIC. http://www.acessoainfor macao.gov.br/sistema/Pedido/ConsultaPedido. aspx, acessado em 15/Out/2012), como facultado pela Lei no 12.527, de 18 de novembro de $201122,227.178 .124$ unidades farmacotécnicas foram obtidas pelos beneficiários do Programa no Município do Rio de Janeiro em 2011, correspondendo a um gasto de $\mathrm{R} \$ 63.372 .116,81$, pagos por este Ministério às farmácias participantes. De que forma a entrada do Programa no município se correlaciona com a evolução nos gastos da SMS-RJ é algo por ser estudado no futuro, mas é possível especular que possa estar repercutindo de alguma forma nos dispêndios com medicamentos do ente municipal.

Outro aspecto importante de ser evocado reside na proporção entre as despesas com aquisições de medicamentos e os gastos municipais em saúde, que foram significativamente mais baixos nos últimos três anos que no restante do período. Em 2011, os gastos com medicamentos em relação ao orçamento total de saúde do Rio de Janeiro corresponderam a apenas 1,8\%, muito inferior a 2006, quando chegaram a representar 
aproximadamente $4,5 \%$. Isso aconteceu mesmo com o crescimento dos orçamentos municipais para a saúde, que têm se elevado desde 2006.

Caliari \& Ruiz 21 analisaram o gasto governamental real total e per capita com medicamentos no período entre 2002-2006. Os gastos por habitante totais cresceram a uma taxa média de $18 \%$, existindo aumento por parte dos estados e municípios (32\% e $74 \%$, respectivamente), porém menores que a elevação dos gastos da União (197\%). No caso dos municípios, os gastos/habitante ascenderam de $\mathrm{R} \$ 2,78$ para $\mathrm{R} \$ 4,84$, no final do intervalo examinado. Quanto à relação despesas com medicamentos sobre despesas totais de saúde, o trabalho não traz dados especificados por município, mas para o Estado do Rio de Janeiro houve crescimento da ordem de 6,29\%.

Vieira \& Zucchi 23 buscaram analisar, para o ano de 2009, a aplicação direta de recursos financeiros na aquisição de medicamentos no SUS pela União, estados e municípios. Estados e o Distrito Federal foram os principais executores, respondendo por $47,1 \%$ do valor total liquidado no SUS (R\$ 8,9 bilhões). A média de gastos per capita dos estados foi de R\$22,00 habitante/ano, com o Estado do Rio de Janeiro situado abaixo desta ( $\mathrm{R} \$ 18,29)$. Há diferenças entre os gastos municipais conforme a região e o porte. A média do gasto per capita daqueles com até cinco mil habitantes foi 3,9 vezes maior que dos com população superior a 500 mil habitantes, em decorrência possivelmente do maior poder de negociação e escala de compras dos municípios maiores.

Em relação ao lócus predominante de uso dos medicamentos (Tabela 2), pode-se verificar que houve uma inversão ao longo do período, com medicamentos vinculados à atenção primária em saúde atingindo, em 2010 , a quase $70 \%$ do total. Esta inversão nos gastos corrigidos é ainda mais relevante se considerado que parte significativa dos fármacos empregados na atenção primária em saúde é, usualmente, de produtos sem valoração de patentes, com tecnologia já difundida e presença de genéricos no mercado e, portanto, com baixo valor unitário e agregado.

Conhecer o desempenho dos gastos municipais da assistência farmacêutica permite orientar suas atividades pela lógica da atenção à saúde, de maneira a aumentar a cobertura qualificada de acesso aos medicamentos, aplicada aos desafios sanitários 24 . Desse modo, a mudança verificada no comportamento do ambiente de gastos traz à tona a discussão em torno da integração da assistência farmacêutica ao modelo de atenção à saúde, que no SUS tem ocorrido por meio do fortalecimento da expansão da atenção primária em saúde, com foco na ESF.
Quanto à distribuição dos gastos de acordo com os subgrupos anatômicos/farmacológicos da classe ATC (Tabela 3), identificou-se contribuição importante de três categorias: anti-infecciosos, sistema cardiovascular e sistema nervoso. Somadas, responderam por quase $56 \%$ do total ( $\mathrm{R} \$ 313.850 .306,82$ em valores corrigidos). Esses subgrupos terapêuticos guardam compatibilidade com o padrão de projeção de carga de doenças no cenário nacional, pois doenças isquêmicas do coração, cerebrovasculares e transtornos depressivos recorrentes constituem-se em causas principais de carga de doença em nosso meio, somadas às doenças infecto-parasitárias, com tendência de declínio, porém ainda muito presentes no cenário nacional 25.

Entre os medicamentos cardiovasculares, destaca-se a participação do anti-hipertensivo hidralazina. Esse medicamento possui patente expirada, um único fabricante (multinacional) e preço unitário elevado, comparado aos demais anti-hipertensivos. Seu valor por unidade farmacotécnica, em 2002, era de $\mathrm{R} \$ 0,08$, ascendendo para $\mathrm{R} \$ 0,15$ em 2011, mesmo com seu volume de aquisição aumentado em quase dez vezes (dados não mostrados). A hidralazina apresenta indicação de uso como segunda ou terceira escolha nos protocolos de atendimento de hipertensão preconizados pela SMS-RJ, usada apenas em hipertensão grave e refratária, emergências hipertensivas, pré-eclampsia grave e eclampsia 26 , e os volumes de gastos podem estar indicando usos não racionais do medicamento.

Chama atenção a queda significativa de gastos referentes aos antineoplásicos, ocorrida a partir de 2005 , de $8 \%$ dos gastos para menos de $0,5 \%$ do total. Até 2004 , a SMS-RJ comprava quantidade significativa de oncológicos para abastecimento, principalmente, dos hospitais que foram refederalizados. Em termos de itens, isto significou a eliminação de aquisição de mais de 50 medicamentos antineoplásicos, em suas várias formas de apresentação.

A expressiva expansão verificada no grupo de medicamentos fitoterápicos pode estar representando a aplicação da Política Nacional de Plantas Medicinais e Fitoterápicos pela SMS-RJ. Há, nesta Secretaria, um Programa de Práticas Integrativas e Complementares, onde se inclui cultivo de certas plantas medicinais em hortas, a manipulação, produção e dispensação de medicamentos fitoterápicos, além da aquisição de produtos industrialmente produzidos.

O financiamento da assistência farmacêutica é apenas uma etapa, que vai muito além dos valores ou processo de aquisição. Nesta lógica, os serviços farmacêuticos precisam estabelecer relacionamentos com seus usuários, favorecendo 
e, muitas vezes, determinando o uso seguro de medicamentos e maximizando seus resultados positivos. Este estudo restringiu-se à análise de montantes e aspectos das aquisições e não deteve seu olhar nestes outros elementos, extremamente relevantes para os processos de cuidado $\mathrm{e}$ de assistência em saúde.

Outra limitação a ser mencionada é que os valores examinados correspondem a empenhos com aquisição de medicamentos pela SMS-RJ. Pode ocorrer, entretanto, que eles não guardem uma correlação absolutamente direta com os gastos reais executados e liquidados por esta Secretaria. Por exemplo, o valor empenhado pode corresponder a uma aquisição, por um dado fornecedor, de mil unidades de um dado fármaco, com um valor empenhado de $\mathrm{R} \$ 1.000,00$, mas este só ter efetivamente entregue $90 \%$ deste volume. O valor pago corresponderá, portanto, a $\mathrm{R} \$$ 900,00 e não aos R\$ 1.000,00 inicialmente empenhados, o que pode trazer algum grau de superestimação nos gastos contabilizados.

O estudo concentrou-se no exame dos gastos com compras diretas regulares de medicamentos. Não estão incluídos, portanto, os gastos da SMS-RJ com os processos de judicialização envolvendo fármacos. Trabalho de Bomfim 27 registra que, em 2006, os gastos do gestor do Município do Rio de Janeiro para atender às demandas judiciais para obtenção de medicamentos somaram $\mathrm{R} \$ 8.311 .016,06$. Considerando os gastos correntes diretos da SMS-RJ com medicamentos para este mesmo ano, este montante corresponde a aproximadamente $17 \%$.

Por fim, deve ser registrada a arbitrariedade da definição do ambiente de prevalência de uso dos medicamentos estabelecida nesta pesquisa, que pode vir a enviesar alguns dos resultados obtidos. Entretanto, a mesma tomou por base uma classificação explícita, qual seja a da RENAME 2010, que considera o uso hospitalar como compreendendo o emprego do medicamento em pacientes internados, acompanhados em hospitais-dia ou em tratamento com antineoplásicos. É possível que um produto de maior uso na atenção primária em saúde possa também ser utilizado, ocasionalmente, no tratamento de pacientes hospitalizados, embora se estime que, se tal ocorre, isso se dê sem representatividade significativa de consumo, quando comparados.

\section{Considerações finais}

Medicamentos são insumos vitais para o funcionamento dos sistemas de saúde e precisam ser garantidos através de políticas governamentais ou medidas que assegurem esta ação. Os programas de assistência farmacêutica no SUS são, muitas vezes, a única forma de acesso aos medicamentos para parte importante da população brasileira, mas agregam pressões significativas sobre os recursos públicos destinados à saúde sendo relevante considerar a sustentabilidade dos programas desenvolvidos nesse contexto assistencial.

O trabalho abrangeu período relativamente extenso e importante, onde ocorreu ampliação do processo de descentralização do financiamento da assistência farmacêutica, sendo possível identificar uma tendência de mudança no perfil de gastos com aquisições dos medicamentos no decênio examinado, invertendo-se do ambiente hospitalar para o lócus da Atenção Primária em Saúde, apesar de não ser suficientemente amplo para aferir uma tendência de longo prazo. Essa transição é compatível com a escolha do Ministério da Saúde ao institucionalizar a ESF como política nacional de atenção primária em saúde e como estruturante dos sistemas municipais de saúde, seguindo uma proposta de reorientação no sistema de saúde do Brasil.

Por fim, os resultados apresentados podem contribuir para uma maior responsabilização do município na gestão e aplicação de recursos com medicamentos na atenção primária em saúde. Essa evidência não é trivial, requerendo uma estrutura técnica e administrativa capaz de aplicação correta e eficiente destes recursos na assistência farmacêutica, compatibilizando o caráter técnico-gerencial com ações técnicoassistenciais que garantam, entre outros, o uso racional de medicamentos nos diversos espaços de atenção. 


\section{Resumen}

El estudio analizó los datos de adquisición de medicamentos del Departamento Municipal de Salud de Río de Janeiro, Brasil, entre 2002-2011. Hemos utilizado los datos financieros que contienen información sobre la cantidad total comprada y el precio por unidad. Se calculó el gasto anual per cápita y la proporción porcentual de los costos de medicamentos, frente a los gastos de salud municipal. Los gastos se han ajustado por el Índice de Precio al Consumidor en su concepto Amplio, para compararlos con 2011. Los medicamentos se clasifican de acuerdo con el uso predominante, entorno hospitalario, o de atención primaria en salud y el sistema de la Organización Mundial de la Salud anatómico-terapéutico-químico. Los gastos totales fueron aproximadamente $R \$ 575$ millones, con una reducción de gastos durante el período analizado. Antiinfecciosos y medicamentos para los sistemas cardiovascular y nervioso representaron el 55\% del gasto total, con una transición del hospital a la atención farmacéutica, vinculada a la atención primaria de salud. En este análisis el uso de datos sobre el gasto permitido mejora la gestión y dirección para la corrección de la política municipal.

Gastos en Salud; Gestión en Salud; Servicios Farmacéuticos; Medicamentos

\section{Colaboradores}

R. M. Silva participou da concepção do estudo, análise e interpretação dos dados e redação do artigo. R. Caetano colaborou na concepção do estudo, revisão crítica e aprovação final da versão a ser publicada.

\section{Agradecimentos}

Os autores agradecem à Aline Navega Biz, pela ajuda no cálculo de deflação dos valores relativos às aquisições com medicamentos, e à Secretaria Municipal de Saúde do Rio de Janeiro, pelo acesso facilitado aos dados utilizados no presente estudo.

\section{Referências}

1. Ministério da Saúde. Portaria GM/MS no 3.916 de 30 de outubro de 1998. Dispõe sobre a aprovação da Política Nacional de Medicamentos. Diário Oficial da União 1998; 10 nov.

2. Ministério da Saúde. Portaria GM/MS no 176, de 8 de março de 1999. Estabelece critérios e requisitos para a qualificação dos municípios e estados ao incentivo à Assistência Farmacêutica Básica e define valores a serem transferidos. Diário Oficial da União 199; 11 mar.

3. Ministério da Saúde. Portaria GM/MS no 1.105, de 5 de julho de 2005. Estabelece normas, responsabilidades e recursos a serem aplicados no financiamento da Assistência Farmacêutica na Atenção Básica e define o Elenco Mínimo Obrigatório de Medicamentos nesse nível de atenção à saúde. Diário Oficial da União 2005; 6 jul.
4. Ministério da Saúde. Portaria GM/MS no 2.084, de 26 de outubro de 2005. Estabelece os mecanismos e as responsabilidades para o financiamento da Assistência Farmacêutica na Atenção Básica e dá outras providências. Diário Oficial da União 2005; 28 out.

5. Ministério da Saúde. Portaria no 3.237, de 24 de dezembro de 2007. Aprova as normas de execução e de financiamento da assistência farmacêutica na atenção básica em saúde. Diário Oficial da União 2007; $26 \mathrm{dez}$.

6. Ministério da Saúde. Portaria no 4.217, de 28 de dezembro de 2010. Aprova as normas de financiamento e execução do Componente Básico da Assistência Farmacêutica. Diário Oficial da União 2010; 29 dez. 
7. Ministério da Saúde. Portaria no 204, de 29 de janeiro de 2007. Regulamenta o financiamento e a transferência dos recursos federais para as ações e os serviços de saúde, na forma de blocos de financiamento, com o respectivo monitoramento e controle. Diário Oficial da União 2007; 31 jan.

8. Ministério da Saúde. Relação nacional de medicamentos essenciais: RENAME. 7ạ Ed. Brasília: Ministério da Saúde; 2010. (Série B. Textos Básicos de Saúde).

9. Pinto LF. Clínicas da família na cidade do Rio de Janeiro: a expansão da atenção primária em saúde em questão. In: Anais do 9o Congresso Nacional da Rede Unida; 2010. http://www.redeunida.org.br/ congressos-anteriores (acessado em 20/Jul/2012).

10. Justo AM. O processo de intervenção federal no Município do Rio de Janeiro: possibilidades de inovação na gestão e a condição dos recursos humanos nos hospitais refederalizados [Dissertação de Mestrado]. Rio de Janeiro: Escola Nacional de Saúde Pública Sergio Arouca, Fundação Oswaldo Cruz; 2008.

11. Brasil. Decreto oo 5.392, de 10 de março de 2005 . Declara estado de calamidade pública no setor hospitalar do Sistema Único de Saúde no Município do Rio de Janeiro, e dá outras providências. Diário Oficial da União 2005; 11 mar.

12. Assessoria de Assistência Farmacêutica, Secretaria Municipal de Saúde e Defesa Civil, Prefeitura Municipal do Rio de Janeiro. Manual de instruções, diretrizes e procedimentos operacionais da assistência farmacêutica. http://subpav.org/downlo ad/assfarm/assfarm_Diretrizes_AF_na_SMSDC_ SUBPAV.pdf (acessado em 22/Jan/2014).

13. WHO Collaborating Centre for Drug Statistics Methodology. Anatomical-Therapeutic-Chemical classification index. Oslo: Norwegian Institute of Public Health; 2009. http://www.whocc.no/atc_ ddd_index/ (acessado em 10/Jul/2012).

14. Brasil. Lei no 10.742, de 6 de outubro de 2003. Define normas de regulação para o setor farmacêutico, cria a Câmara de Regulação do Mercado de Medicamentos - CMED e altera a Lei no 6.360, de 23 de setembro de 1976, e dá outras providências. Diário Oficial da União 2003; 07 out.

15. Vieira FS, Mendes ACR. Evolução dos gastos com medicamentos: crescimento que preocupa. In: Anais do VIII Encontro da Associação Brasileira de Economia da Saúde. São Paulo: Pontifícia Universidade Católica de São Paulo; 2007. http://abres brasil.org.br/trabalhos/evolucao-dos-gastoscom-medicamentos-crescimento-que-preocupa. html (acessado em 22/Jan/2014).

16. Vieira FS. Gastos do Ministério da Saúde com medicamentos: tendência dos programas de 2002 a 2007. Rev Saúde Pública 2009; 43:674-81.
17. Carias CM, Vieira FB, Giordano CV, Zucchi P. Medicamentos de dispensação excepcional: histórico e gastos do Ministério da Saúde do Brasil. Rev Saúde Pública 2011; 45:233-40.

18. Vieira FS. Assistência farmacêutica no sistema público de saúde no Brasil. Rev Panam Salud Pública 2010; 27:149-56.

19. Aurea AP, Magalhães LCG, Garcia LP, Santos CF, Almeida RF. Programas de assistência farmacêutica do Governo Federal: estrutura atual, evolução dos gastos com medicamentos e primeiras evidências de sua eficiência, 2005-2008. Brasília: Instituto de Pesquisa Econômica Aplicada; 2011.

20. Presidência da República. Decreto no 5.090, de 20 de maio de 2004. Regulamenta a Lei no 10.858 , de 13 de abril de 2004, e institui o programa "Farmácia Popular do Brasil", e dá outras providências. Diário Oficial da União 2004; 21 mai.

21. Caliari T, Ruiz RM. Os gastos públicos com medicamentos no período de 2002 a 2006. Rev Adm Saúde 2010; 12:167-76.

22. Presidência da República. Lei no 12.527 , de 18 de novembro de 2011. Regula o acesso a informações previsto no inciso XXXIII do art. 5o, no inciso II do $\$ 30$ do art. 37 e no $\$ 2$ o do art. 216 da Constituição Federal; altera a Lei no 8.112 , de 11 de dezembro de 1990; revoga a Lei no 11.111, de 5 de maio de 2005, e dispositivos da Lei no 8.159, de 8 de janeiro de 1991; e dá outras providências. Diário Oficial da União 2011; 18 nov.

23. Vieira FS, Zucchi P. Aplicações diretas para aquisição de medicamentos no Sistema Único de Saúde. Rev Saúde Pública 2011; 45:906-13.

24. Conselho Nacional de Secretários de Saúde. Assistência farmacêutica no SUS. v. 7. Brasília: Conselho Nacional de Secretários de Saúde; 2007.

25. Leite IC, Beltrão KI, Rodrigues RN, Valente JG, Campos MR, Schramm JM. Projeção da carga de doença no Brasil (1998-2013). In: Buss PM, Temporão JG, Carvalheiro JR, organizadores. Vacinas, soros \& imunizações no Brasil. Rio de Janeiro: Editora Fiocruz; 2005. p. 51-65.

26. Secretaria Municipal de Saúde e Defesa Civil. Hipertensão: protocolos de atendimento. http:// www.rio.rj.gov.br/web/smsdc/exibeConteudo? article-id=124760 (acessado em 01/Ago/2012)

27. Bomfim RLD. Agenda única de saúde: a busca do acesso universal e a garantia do direito à saúde [Tese de Doutorado]. Rio de Janeiro: Instituto de Medicina Social, Universidade do Estado do Rio de Janeiro; 2008.

Recebido em 06/Ago/2012

Versão final reapresentada em 06/Mai/2013 Aprovado em 12/Dez/2013 